\title{
A priori bounds and existence of non-real eigenvalues of indefinite Sturm-Liouville problems
}

\author{
Jiangang Qi and Shaozhu Chen ${ }^{1}$
}

\begin{abstract}
The present paper gives a priori bounds on the possible non-real eigenvalues of regular indefinite Sturm-Liouville problems and obtains sufficient conditions for such problems to admit non-real eigenvalues.
\end{abstract}

Mathematics Subject Classification (2010). 34B24, 34L15, 47B50.

Keywords. A priori bound, non-real eigenvalue, indefinite Sturm-Liouville problem.

\section{Introduction}

The present paper is concerned with the indefinite spectral problem

$$
-y^{\prime \prime}+q y=\lambda w y, y(-1)=y(1)=0, \quad \text { in } L_{|w|}^{2}[-1,1]
$$

under the standing hypothesis that $q$ and $w$ are real-valued functions satisfying

$$
q, w \in L^{1}[-1,1], \quad w(x) \neq 0 \text { a.e. on }[-1,1]
$$

and $w(x)$ changes sign on $[-1,1]$. The indefinite problem (1.1) has discrete, real eigenvalues, unbounded from both below and above, and may also admit non-real eigenvalues. Such problems occur in certain physical models, particularly in transport theory and statistical physics. The indefinite nature of the problem was noticed by Haupt [9] and Richardson [12] at the beginning of the last century. For a review of the early work in this direction, see [11].

\footnotetext{
${ }^{1}$ This research was partially supported by the NSF of China (Grant 11271229), the NSF of Shandong Province (Grants ZR2012AM002 and ZR2011AQ002) and the Teaching Research and Teaching Reform Project of Shandong University, Weihai (Grant A201005).
} 
As a simple example of (1.1), the Richardson problem [13]

$$
-y^{\prime \prime}-\mu y=\lambda \operatorname{sgn}(x) y, \quad x \in[-1,1], \quad y(-1)=0=y(1)
$$

was studied by many authors, such as Turyn [14], Atkinson and Jabon [1], Fleckinger and Mingarelli [8], and P. Binding and H. Volkmer [6]. For the indefinite problem (1.1), non-real eigenvalues might appear only if the corresponding right-definite problem

$$
-y^{\prime \prime}+q y=\lambda|w| y, y(-1)=y(1)=0 \text { in } L_{|w|}^{2}[-1,1]
$$

has negative eigenvalues, namely, here holds the following result.

Proposition 1.1 ([10], Theorem 2, p. 523, and [7], Corollary 1.7). If problem (1.4) has $n$ negative eigenvalues, then problem (1.1) has at most $2 n$ non-real eigenvalues.

Although the upper bound given in Proposition 1.1 is sharp (see [12] and [2]), determining a priori bounds and the exact number of non-real eigenvalues are still difficult and interesting open problems in Sturm-Liouville theory (see [11] and [15], p. 126). Recently, by means of the operator theory in Krein spaces, Behrndt, Katatbeh, and Trunk [2], Theorem 2.3 and Corollary 2.4, have given sufficient conditions for the existence of non-real eigenvalues of the singular indefinite Sturm-Liouville operator

$$
(A f)(x) \stackrel{\text { def }}{=} \operatorname{sgn}(x)\left(-f^{\prime \prime}(x)+V(x) f(x)\right)=\lambda f(x), \quad x \in \mathbb{R},
$$

and if $V \in L^{\infty}(\mathbb{R})$, Behrndt, Philipp and Trunk [3], Theorem 4.2, have obtained explicit bounds on the non-real eigenvalues of (1.5) in terms of $V$.

In the present paper, we will first obtain a priori bounds for possible non-real eigenvalues and then find sufficient conditions for the existence of non-real eigenvalues of (1.1). These results will answer or partially answer several open problems posed in [11]. We state these results in this section and prove them in Sections 2 and 3 .

Denote by $\|\cdot\|_{p}$ the norm of the space $L^{p}[-1,1]$ and by $\|\cdot\|_{C}$ the maximum norm of $C[-1,1]$. If $x w(x)>0$ a.e. on $[-1,1]$, we set

$$
S_{1}(\varepsilon)=\{x \in[-1,1]: x w(x)<\varepsilon\}, \quad m_{1}(\varepsilon)=\operatorname{mes} S_{1}(\varepsilon) .
$$

If $w \in A C_{\text {loc }}[-1,1], w^{\prime} \in L^{2}[-1,1]$, we set

$$
S_{2}(\varepsilon)=\left\{x \in[-1,1]: w^{2}(x)<\varepsilon\right\}, \quad m_{2}(\varepsilon)=\operatorname{mes} S_{2}(\varepsilon) .
$$

A value of $x$ about which $w(x)$ changes its sign will be called a turning point [10]. If $w(x)$ has only one turning point, we will obtain the following a priori bounds for possible non-real eigenvalues. 
Theorem 1.2. Suppose that $\lambda$ exists and that it is a non-real eigenvalue of (1.1). If $x w(x)>0$ a.e. on $[-1,1]$, then

$$
|\operatorname{Re} \lambda| \leq \frac{4}{\varepsilon_{1}}\left(\left\|q_{-}\right\|_{1}+4\left\|q_{-}\right\|_{1}^{2}\right), \quad|\operatorname{Im} \lambda| \leq \frac{4}{\varepsilon_{1}}\left\|q_{-}\right\|_{1},
$$

where $\varepsilon_{1}>0$ satisfies $8\left\|q_{-}\right\|_{1}^{2} m_{1}\left(\varepsilon_{1}\right)<1$ and $q_{-}(x)=-\min \{0, q(x)\}$.

In the case where $w(x)$ is allowed to have more turning points, we will obtain the following result.

Theorem 1.3. Suppose that $\lambda$ exists and that it is a non-real eigenvalue of (1.1). If $w \in A C[-1,1]$ and $w^{\prime} \in L^{2}[-1,1]$, then

$$
|\operatorname{Re} \lambda| \leq \frac{8}{\varepsilon_{2}}\left\|q_{-}\right\|_{1}^{2}\left(3\|w\|_{C}+\left\|w^{\prime}\right\|_{2}\right), \quad|\operatorname{Im} \lambda| \leq \frac{8}{\varepsilon_{2}}\left\|w^{\prime}\right\|_{2}\left\|q_{-}\right\|_{1}^{2},
$$

where $\varepsilon_{2}>0$ is chosen such that $8\left\|q_{-}\right\|_{1}^{2} m_{2}\left(\varepsilon_{2}\right)<1$.

In the particular case where $q \geq 0$, we see by Theorems 1.2 and 1.3 that (1.1) has no any non-real eigenvalues, which is in accordance with the conclusion in Proposition 1.1 since now (1.4) does not have any negative eigenvalues.

In what follows, we impose the symmetry conditions on $q$ and $w$, namely,

$$
q(x)=q(-x) \text { and } \quad w(-x)=-w(x) .
$$

In this case, more accurate a priori bounds on imaginary eigenvalues can be found if $q$ is bounded below and $w$ keeps away from zero.

Theorem 1.4. Suppose that $(1.10)$ holds and $x w(x)>0$ a.e. on $[-1,1]$. If, for some $q_{0}<0$ and $w_{0}>0$,

$$
q(x) \geq q_{0},|w(x)| \geq w_{0} \text { a.e. } x \in[-1,1],
$$

then for any possible pure imaginary eigenvalue $\lambda$ of (1.1), there holds

$$
|\operatorname{Im} \lambda| \leq \frac{4\left(-q_{0}\right)^{3 / 2}}{w_{0}}
$$

In view of (1.10), using the spectral theory of operators in Krein spaces, we obtain an existence result for non-real eigenvalues of the indefinite problem (1.1).

Theorem 1.5. Let (1.10) be fulfilled. If the eigenvalue problem

$$
-y^{\prime \prime}+q(x) y=\lambda y, \quad y(-1)=y(1)=0
$$

has one negative eigenvalue and the rest eigenvalues are all positive, then (1.1) has exactly two purely imaginary eigenvalues. 
Immediate consequences of Proposition 1.1, Theorems 1.4, and Theorem 1.5 are the existence and bounds for non-real eigenvalues of Richardson problem.

Corollary 1.6. For $\mu \in\left(\frac{\pi^{2}}{4}, \pi^{2}\right)$, the Richardson eigenvalue problem (1.3) has exactly two purely imaginary eigenvalues whose moduli are bounded by $4 \mu^{3 / 2}$.

Remark 1. Theorems 1.2 and 1.5 can be generalized to the problem

$$
\left\{\begin{array}{l}
-\left(p(x) y^{\prime}\right)^{\prime}+q(x) y=\lambda w(x) y, \\
\alpha_{1} y(-1)+\beta_{1} y^{\prime}(-1)=0 \\
\alpha_{2} y(1)+\beta_{2} y^{\prime}(1)=0
\end{array}\right.
$$

where $p(x)>0$ a.e. on $[-1,1], 1 / p \in L^{1}[-1,1], \alpha_{j}, \beta_{j} \in \mathbb{R}$ for $j=1,2$ and $\alpha_{1} \beta_{2}+\alpha_{2} \beta_{1}=0$, but we do not pursue this here.

Acknowledgments. The authors gratefully thank the referee for his or her helpful suggestions which lead to the improvement of the result in and simplification of the proof of Theorem 1.5.

\section{A priori bounds of non-real eigenvalues}

In this section we will prove Theorems 1.2, 1.3, and 1.4.

Proof of Theorem 1.2. Let $\lambda$ be a non-real eigenvalue of $(1.1)$ and $\varphi(x)$ the corresponding eigenfunction with $\|\varphi\|_{2}=1$. Multiplying both sides of $-\varphi^{\prime \prime}+q \varphi=\lambda w \varphi$ by $\bar{\varphi}$ and integrating over the interval $[x, 1]$ we have

$$
\left(\varphi^{\prime} \bar{\varphi}\right)(x)+\int_{x}^{1}\left|\varphi^{\prime}\right|^{2}+\int_{x}^{1} q|\varphi|^{2}=\lambda \int_{x}^{1} w|\varphi|^{2} .
$$

Separating the real and imaginary parts of both sides of (2.1) yields

$$
\begin{aligned}
& \operatorname{Re} \lambda \int_{x}^{1} w|\varphi|^{2}=\operatorname{Re}\left(\varphi^{\prime} \bar{\varphi}\right)(x)+\int_{x}^{1}\left|\varphi^{\prime}\right|^{2}+\int_{x}^{1} q|\varphi|^{2}, \\
& \operatorname{Im} \lambda \int_{x}^{1} w|\varphi|^{2}=\operatorname{Im}\left(\varphi^{\prime} \bar{\varphi}\right)(x) .
\end{aligned}
$$

We will use (2.2) and (2.3) to estimate $\operatorname{Re} \lambda$ and $\operatorname{Im} \lambda$. To do this, let $x=-1$ in (2.3). From $\operatorname{Im} \lambda \neq 0$ and $\varphi(-1)=0$, we have $\int_{-1}^{1} w|\varphi|^{2}=0$, and hence, by (2.2),

$$
\int_{-1}^{1}\left(\left|\varphi^{\prime}\right|^{2}+q|\varphi|^{2}\right)=0
$$


Set

$$
Q(x)=\int_{-1}^{x} q_{-}(t) \mathrm{d} t
$$

Then $\max |Q(x)| \leq\left\|q_{-}\right\|_{1}$ and

$$
\int_{-1}^{1} q_{-}(x)|\varphi(x)|^{2} \mathrm{~d} x=\int_{-1}^{1} Q^{\prime}(x)|\varphi(x)|^{2} \mathrm{~d} x-2 \operatorname{Re}\left(\int_{-1}^{1} Q(x) \varphi^{\prime}(x) \overline{\varphi(x)} \mathrm{d} x\right),
$$

which, together with $\|\varphi\|_{2}=1$, yields that

$$
\begin{aligned}
\int_{-1}^{1} q_{-}|\varphi|^{2} & \leq 2\left\|q_{-}\right\|_{1} \int_{-1}^{1}\left|\varphi^{\prime}\right||\varphi| \\
& \leq 2\left\|q_{-}\right\|_{1}\left\|\varphi^{\prime}\right\|_{2} \leq 2\left\|q_{-}\right\|_{1}^{2}+\frac{1}{2}\left\|\varphi^{\prime}\right\|_{2}^{2}
\end{aligned}
$$

Then, from (2.4), we get

$$
\left\|\varphi^{\prime}\right\|_{2}^{2} \leq 4\left\|q_{-}\right\|_{1}^{2}, \int_{-1}^{1} q_{-}|\varphi|^{2} \leq 4\left\|q_{-}\right\|_{1}^{2}
$$

From $\varphi(x)=\int_{-1}^{x} \varphi^{\prime}(t) \mathrm{d} t$, by Cauchy-Schwarz inequality, we have

$$
|\varphi(x)|^{2}=\left|\int_{-1}^{x} \varphi^{\prime}(t) \mathrm{d} t\right|^{2} \leq(x+1) \int_{-1}^{x}\left|\varphi^{\prime}(t)\right|^{2} \mathrm{~d} t \leq \int_{-1}^{0}\left|\varphi^{\prime}\right|^{2} \leq\left\|\varphi^{\prime}\right\|_{2}^{2}
$$

for $-1 \leq x \leq 0$. From $\varphi(x)=-\int_{x}^{1} \varphi^{\prime}(t) \mathrm{d} t$, one similarly proves $|\varphi(x)|^{2} \leq\left\|\varphi^{\prime}\right\|_{2}^{2}$ for $x \in[0,1]$, and so,

$$
|\varphi(x)|^{2} \leq\left\|\varphi^{\prime}\right\|_{2}^{2}, \quad x \in[-1,1]
$$

Since $x w(x)>0$, a.e. on $[-1,1]$, one can find $\varepsilon_{1}>0$ such that $8\left\|q_{-}\right\|_{1}^{2} m_{1}\left(\varepsilon_{1}\right)<1$, where $m_{1}(\varepsilon)$ is defined in (1.6). Using $\int_{-1}^{1} w|\varphi|^{2}=0$, from (2.6) and (2.7), we have

$$
\begin{aligned}
\int_{-1}^{1} \int_{x}^{1} w(t)|\varphi(t)|^{2} \mathrm{~d} t \mathrm{~d} x & =\int_{-1}^{1} x w(x)|\varphi(x)|^{2} \mathrm{~d} x \\
& \geq \varepsilon_{1}\left(\int_{-1}^{1}|\varphi(x)|^{2} \mathrm{~d} x-\int_{S_{1}\left(\varepsilon_{1}\right)}|\varphi(x)|^{2} \mathrm{~d} x\right) \\
& \geq \varepsilon_{1}\left[1-4\left\|q_{-}\right\|_{1}^{2} m_{1}\left(\varepsilon_{1}\right)\right] \\
& \geq \frac{\varepsilon_{1}}{2}
\end{aligned}
$$


Set

$$
q_{+}(x)=\max \{0, q(x)\}
$$

Then $q=q_{+}-q_{-}$and $|q|=q_{+}+q_{-}=q+2 q_{-}$. Repeatedly using (2.4), we have

$$
\begin{aligned}
\left|\int_{-1}^{1} \int_{x}^{1}\left(\left|\varphi^{\prime}\right|^{2}+q|\varphi|^{2}\right) \mathrm{d} t \mathrm{~d} x\right| & =\left|\int_{-1}^{1} x\left(\left|\varphi^{\prime}\right|^{2}+q|\varphi|^{2}\right) \mathrm{d} x\right| \\
& \leq \int_{-1}^{1}\left(\left|\varphi^{\prime}\right|^{2}+q|\varphi|^{2}+2 q-|\varphi|^{2}\right) \mathrm{d} x \\
& =2 \int_{-1}^{1} q_{-}|\varphi|^{2} \mathrm{~d} x
\end{aligned}
$$

Now, by (2.6), the integration of (2.2) gives

$$
\begin{aligned}
|\operatorname{Re} \lambda| \int_{-1}^{1} \int_{x}^{1} w|\varphi|^{2} & =\left|\int_{-1}^{1} \operatorname{Re}\left(\varphi^{\prime} \bar{\varphi}\right) \mathrm{d} x+\int_{-1}^{1} \int_{x}^{1}\left(\left|\varphi^{\prime}\right|^{2}+q|\varphi|^{2}\right) \mathrm{d} t \mathrm{~d} x\right| \\
& \leq\left\|\varphi^{\prime}\right\|_{2}+2 \int_{-1}^{1} q_{-}|\varphi|^{2} \mathrm{~d} x \leq 2\left\|q_{-}\right\|_{1}+8\left\|q_{-}\right\|_{1}^{2} .
\end{aligned}
$$

Therefore, in view of (2.8), we conclude that

$$
|\operatorname{Re} \lambda| \leq \frac{4}{\varepsilon_{1}}\left(\left\|q_{-}\right\|_{1}+4\left\|q_{-}\right\|_{1}^{2}\right) .
$$

Moreover, integrating (2.3) and using (2.8) and (2.6), we have

$$
\frac{\varepsilon_{1}}{2}|\operatorname{Im} \lambda| \leq|\operatorname{Im} \lambda| \int_{-1}^{1} \int_{x}^{1} w|\varphi|^{2}=\left|\int_{-1}^{1} \operatorname{Im}\left(\varphi^{\prime} \bar{\varphi}\right)\right| \leq\left\|\varphi^{\prime}\right\|_{2} \leq 2\left\|q_{-}\right\|_{1},
$$

and (1.8) follows immediately. This completes the proof of Theorem 1.2.

Proof of Theorem 1.3. Let $\lambda$ be a non-real eigenvalue of (1.1) and $\varphi$ the corresponding eigenfunction with $\|\varphi\|_{2}=1$. In this case we still can make use of (2.1), (2.2), and (2.3). From (2.3), since $\operatorname{Im} \lambda \neq 0$, one sees that $\int_{-1}^{1} w|\varphi(x)|^{2} \mathrm{~d} x=0$. Thus, (2.4), (2.6), and (2.7) hold, and, in particular,

$$
|\varphi(x)|^{2} \leq\left\|\varphi^{\prime}\right\|_{2}^{2}, \quad x \in[-1,1], \quad\left\|\varphi^{\prime}\right\|_{2}^{2} \leq \int_{-1}^{1} q_{-}|\varphi|^{2} \leq 4\left\|q_{-}\right\|_{1}^{2} .
$$

Multiplying $-\varphi^{\prime \prime}+q \varphi=\lambda w \varphi$ by $w \bar{\varphi}$ and integrating by parts, we get

$$
\int_{-1}^{1} w\left|\varphi^{\prime}\right|^{2}+\int_{-1}^{1} w^{\prime} \varphi^{\prime} \bar{\varphi}+\int_{-1}^{1} w q|\varphi|^{2}=\lambda \int_{-1}^{1} w^{2}|\varphi|^{2}
$$


Separating the real and imaginary parts of the both sides of (2.12) yields

$$
\begin{aligned}
& \operatorname{Re} \lambda \int_{-1}^{1} w^{2}|\varphi|^{2}=\operatorname{Re}\left(\int_{-1}^{1} w^{\prime} \varphi^{\prime} \bar{\varphi}\right)+\int_{-1}^{1} w\left(\left|\varphi^{\prime}\right|^{2}+q|\varphi|^{2}\right), \\
& \operatorname{Im} \lambda \int_{-1}^{1} w^{2}|\varphi|^{2}=\operatorname{Im}\left(\int_{-1}^{1} w^{\prime} \varphi^{\prime} \bar{\varphi}\right) .
\end{aligned}
$$

Now, using (2.11), $|q|=q+2 q_{-}$and

$$
\int_{-1}^{1} q|\varphi|^{2}=-\int_{-1}^{1}\left|\varphi^{\prime}\right|^{2}<0,
$$

we obtain

$$
\begin{aligned}
\left.\left|\int_{-1}^{1} w\right| \varphi^{\prime}\right|^{2} \mid & \leq\|w\|_{C}\left\|\varphi^{\prime}\right\|_{2}^{2} \leq 4\|w\|_{C}\left\|q_{-}\right\|_{1}^{2}, \\
\left.\left|\int_{-1}^{1} w q\right| \varphi\right|^{2} \mid & \leq\|w\|_{C} \int_{-1}^{1}\left|q\left\|\left.\varphi\right|^{2} \leq 8\right\| w\left\|_{C}\right\| q_{-} \|_{1}^{2},\right. \\
\left|\int_{-1}^{1} w^{\prime} \varphi^{\prime} \bar{\varphi}\right| & \leq\left\|\varphi^{\prime}\right\|_{2}\left\|w^{\prime}\right\|_{2}\left\|\varphi^{\prime}\right\|_{2} \leq 4\left\|w^{\prime}\right\|_{2}\left\|q_{-}\right\|_{1}^{2} .
\end{aligned}
$$

Recall that $m_{2}\left(\varepsilon_{2}\right)=$ mes $S_{2}\left(\varepsilon_{2}\right)$ defined in (1.7) and $w^{2}(x) \geq \varepsilon_{2}$ on the set $\Omega\left(\varepsilon_{2}\right) \stackrel{\text { def }}{=}[-1,1] \backslash S_{2}\left(\varepsilon_{2}\right)$. Then $8\|q-\|_{1}^{2} m\left(\varepsilon_{2}\right)<1$ yields that

$$
\begin{aligned}
\int_{-1}^{1} w^{2}(x)|\varphi(x)|^{2} \mathrm{~d} x & \geq \varepsilon_{2} \int_{\Omega\left(\varepsilon_{2}\right)}|\varphi|^{2} \\
& =\varepsilon_{2}\left(1-\int_{S\left(\varepsilon_{2}\right)}|\varphi|^{2}\right) \\
& \geq \varepsilon_{2}\left(1-4\|q-\|_{1}^{2} m\left(\varepsilon_{2}\right)\right) \\
& \geq \frac{\varepsilon_{2}}{2}
\end{aligned}
$$

which, together with (2.13), (2.14), and (2.15), gives (1.9) and completes the proof.

Under conditions (1.2) and (1.10), it is easy to see that if $\lambda \in \mathbb{C}$ is an eigenvalue of (1.1) with an eigenfunction $\varphi$, then $-\bar{\lambda}$ is an eigenvalue of (1.1) with the eigenfunction $\overline{\varphi(-\cdot)}$. Thus, if $\lambda=\mathrm{i} \alpha$ with $\alpha \in \mathbb{R}$, then $\overline{\varphi(-x)}=C \varphi(x)$ for some $C \neq 0$ since the geometric multiplicity is one. Then it follows that $|C|=1$ from $\overline{\varphi(0)}=C \varphi(0), \overline{\varphi^{\prime}(0)}=-C \varphi^{\prime}(0)$, and $|\varphi(0)|+\left|\varphi^{\prime}(0)\right| \neq 0$. To sum up, we have the following result. 
Lemma 2.1. Let (1.2) and (1.10) hold. If $\lambda \in \mathbb{C}$ is an eigenvalue of (1.1) with an eigenfunction $\varphi$, then $-\bar{\lambda}$ is an eigenvalue of (1.1) with the eigenfunction $\overline{\varphi(-\cdot)}$. In particular, if $\lambda=\mathrm{i} \alpha$ with $\alpha \in \mathbb{R}$ and $\alpha \neq 0$, then $\overline{\varphi(-\cdot)}=C \varphi$ for some $C \in \mathbb{C}$ with $|C|=1$.

Proof of Theorem 1.4. Let $\varphi$ be an eigenfunction corresponding to $\lambda=\mathrm{i} \alpha$ with $\|\varphi\|_{2}=1$. It follows from Lemma 2.1 that there exists an $\omega \in[0,2 \pi)$ such that $\overline{\varphi(-x)}=\mathrm{e}^{\mathrm{i} \omega} \varphi(x)$ and $-\overline{\varphi^{\prime}(-x)}=\mathrm{e}^{\mathrm{i} \omega} \varphi^{\prime}(x)$. So, $|\varphi(x)|$ and $\left|\varphi^{\prime}(x)\right|$ are even functions. We see that (2.1)-(2.4) hold for this $\varphi$. Similarly to (2.7), we have

$$
\begin{aligned}
|\varphi(x)|^{2} & \leq(x+1) \int_{-1}^{x}\left|\varphi^{\prime}(t)\right|^{2} \mathrm{~d} t \\
& \leq \int_{-1}^{0}\left|\varphi^{\prime}(t)\right|^{2} \mathrm{~d} t=\frac{1}{2}\left\|\varphi^{\prime}\right\|_{2}^{2}, \quad x \in[-1,0],
\end{aligned}
$$

since $\left|\varphi^{\prime}(x)\right|$ is even. Actually, (2.17) is true for $x \in[-1,1]$ since $|\varphi(x)|$ is even.

Since $q(x) \geq q_{0}$ on $[-1,1]$, it follows from (2.4) and $\|\varphi\|_{2}=1$ that

$$
\left\|\varphi^{\prime}\right\|_{2}^{2}=-\int_{-1}^{1} q|\varphi|^{2} \leq-q_{0},
$$

and then the integration of (2.3) produces

$$
\left.|\operatorname{Im} \lambda|\left|\int_{-1}^{1} \int_{x}^{1} w\right| \varphi\right|^{2}|=| \int_{-1}^{1} \operatorname{Im}\left(\varphi^{\prime} \bar{\varphi}\right) \mid \leq\left\|\varphi^{\prime}\right\|_{2} \leq\left(-q_{0}\right)^{1 / 2} .
$$

Let $\delta=1 /\left(-2 q_{0}\right)$. By (2.17), we have $1=\int_{-1}^{1}|\varphi|^{2} \leq\left\|\varphi^{\prime}\right\|_{2}^{2} \leq-q_{0}$ and

$$
\begin{aligned}
\left.\left|\int_{-1}^{1} \int_{x}^{1} w\right| \varphi\right|^{2} \mid & =\int_{-1}^{1} x w(x)|\varphi|^{2} \mathrm{~d} x \\
& \geq w_{0} \int_{-1}^{1}|x||\varphi|^{2} \mathrm{~d} x \\
& \geq w_{0} \delta \int_{|x| \geq \delta}|\varphi|^{2}=w_{0} \delta\left(1-\int_{-\delta}^{\delta}|\varphi|^{2}\right) \\
& \geq w_{0} \delta\left(1-\delta\left(-q_{0}\right)\right) \\
& =-\frac{w_{0}}{4 q_{0}} .
\end{aligned}
$$

Now, (1.12) follows from (2.18) and (2.19). The proof is complete. 


\section{Existence of non-real eigenvalues}

In this section we prove Theorem 1.5 and in the proof we will use the following result which was proved, e.g., in [7] and [5].

Lemma 3.1 ([7], Proposition 2.6). If $w_{j} \in L^{1}[-1,1]$ and $w_{j}(x)>0$ a.e. on $[-1,1]$ for $j=1,2$, then the two eigenvalue problems

$$
-y^{\prime \prime}+q(x) y=\lambda w_{j}(x) y, \quad y(-1)=y(1)=0, \quad j=1,2
$$

have the same number of negative eigenvalues.

Let $K$ be the Krein space $L_{|w|}^{2}[-1,1]$, equipped with the indefinite inner product

$$
[f, g]=\int_{-1}^{1} f(x) \overline{g(x)} w(x) \mathrm{d} x, f, g \in L_{|w|}^{2}[-1,1]
$$

and $T$ a self-adjoint operator in $K$ with domain $\mathscr{D}(T)$; see [4], [2], and [7]. We say that the operator $T$ has $k$ negative squares, $k \in \mathbb{N}_{0}$, if there exists a $k$-dimensional subspace $X$ of $K$ in $\mathscr{D}(T)$ such that $[T f, f]<0$ if $f \in X$ and $f \neq 0$, but no $(k+1)$-dimensional subspace with this property.

Proof of Theorem 1.5. Let $A$ and $B$ be the operators associated with $-y^{\prime \prime}+q(x) y=$ $\lambda w(x) y$ and $-y^{\prime \prime}+q(x) y=\lambda|w(x)| y$ with the Dirichlet boundary conditions, respectively. Then $B$ is self-adjoint with respect to the definite inner product

$$
(f, g)=\int_{-1}^{1} f(x) \overline{g(x)}|w(x)| \mathrm{d} x, f, g \in L_{|w|}^{2}[-1,1]
$$

and $A$ is self-adjoint with respect to the indefinite inner product (3.2).

It follows from Lemma 3.1 and the assumption in Theorem 1.5 that $B$ has one negative eigenvalue and the rest are positive, and hence, $A$ has exactly one negative square since $[A f, f]=(B f, f)$ and 0 is a resolvent point of $A$. It is well known (see, e.g., [7], Proposition 1.5, or [4], Theorem 3.1) that this implies the existence of exactly one eigenvalue $\lambda$ of (1.1) in $\mathbb{R}$ or the upper half-plane $\mathbb{C}^{+}$and that if $\lambda \in \mathbb{R}$ with eigenfunction $\varphi$ then $[A \varphi, \varphi]=\lambda[\varphi, \varphi] \leq 0$. Let $\lambda$ be such an eigenvalue with eigenfunction $\varphi$. If $\lambda$ is real, then $-\lambda=-\bar{\lambda}$ is also an eigenvalue with the eigenfunction $\overline{\varphi(-\cdot)}$ by Lemma 2.1 and

$$
-\lambda[\overline{\varphi(-\cdot)}, \overline{\varphi(-\cdot)}]=\lambda[\varphi, \varphi] \leq 0
$$

by the odd symmetry of $w$. Thus, we get that $\lambda$ and $-\lambda$ are two such eigenvalues, which is a contradiction. Since $\lambda \in \mathbb{C}^{+}$implies $-\bar{\lambda} \in \mathbb{C}^{+}$, we see that $\lambda=-\bar{\lambda}$, i.e., $\lambda$ is purely imaginary. The proof of Theorem 1.5 is complete. 


\section{References}

[1] F. Atkinson and D. Jabon, Indefinite Sturm-Liouville problems. In H. Kaper, M.-K. Kwong, and A. Zettl (eds.), Proceedings of the Focused Research Program on Spectral Theory and Boundary Value Problems. Vol. I. Argonne National Laboratory, Mathematics and Computer Science Division, Argonne, IL, 1987, 31-45.

[2] J. Behrndt, Q. Katatbeh and C. Trunk, Non-real eigenvalues of singular indefinite Sturm-Liouville operators. Proc. Amer. Math. Soc. 137 (2009), 3797-3806. MR 2529889 Zbl 1182.47036

[3] J. Behrndt, F. Philipp and C. Trunk, Bounds on the non-real spectrum of differential operators with indefinite weights. Math. Ann. 357 (2013), 185-213. MR 3084346 Zbl 06210502

[4] J. Behrndt and C. Trunk, On the negative squares of indefinite Sturm-Liouville operators. J. Diff. Equations 238 (2007), 491-519. MR 2341434 Zbl 1123.47033

[5] P. Binding and M. Möller, Negativity indices for definite and indefinite Sturm-Liouville problems. Math. Nachr. 283 (2010), 180-192. MR 2604116 Zbl 1193.34054

[6] P. Binding and H. Volkmer, Eigencurves for two-parameter Sturm-Liouville equations. SIAM Review 38 (1996), 27-48. MR 1379040 Zbl 0869.34020

[7] B. Curgus and H. Langer, A Krein space approach to symmetric ordinary differential operators with an indefinite weigth function. J. Diff. Equations 79 (1989), 31-61. MR 0997608 Zbl 0693.34020

[8] J. Fleckinger and A. B. Mingarelli, On the eigenfunctions of non-definite elliptic operators. In I. W. Knowles and R. T. Lewis (eds.), Differential Equations. North-Holland, Amsterdam, 1984, 229-228.

[9] O. Haupt, Über eine methode zum beweis von oszillations theoremen. Math. Ann. 76 (1915), 67-104.

[10] A. B. Mingarelli, Indefinite Sturm-Liouville problems. In W. N. Everitt and B. D. Sleeman (eds.), Ordinary and partial differential equations. Proceedings of the Seventh Conference held at the University of Dundee, Dundee, March 29-April 2, 1982. Lecture Notes in Mathematics 964. Springer Verlag, Berlin etc., 1982, 519-528. MR 0693136 Zbl 0488.00008 (collection)

[11] A. B. Mingarelli, A survey of the regular weighted Sturm-Liouville problem - The non-definite case. In S. T. Xiao and F. Q. Pu (eds.), International workshop on applied differential equations. Proceedings of the workshop held at Tsinghua University, Beijing, June 4-7, 1985. World Scientific, Singapore, 1986, 109-137. MR 0901329 Zbl 0624.34021

[12] R. G. D. Richardson, Theorems of oscillation for two linear differential equations of second order with two parameters. Trans. Amer. Math. Soc. 13 (1912), 22-34. MR 1500902 JFM 43.0400.03

[13] R. G. D. Richardson, Contributions to the study of oscillation properties of the solutions of linear differential equations of the second order. Amer. J. Math. 40 (1918), 283-316. MR 1506360 JFM 46.0698.03

[14] L. Turyn, Sturm-Liouville problems with several parameters. J. Differential Equations 38 (1980), 239-259. MR 0597803 Zbl 0421.34023 
[15] A. Zettl, Sturm-Liouville Theory. Mathematical Surveys and Monographs 121. American Mathematical Society, Providence, RI, 2005. MR 2170950 Zbl 1103.34001

Received December 17, 2012

Jiangang Qi, Department of Mathematics, Shandong University (Weihai), Weihai 264209, P. R. China

E-mail: qjg816@163.com

Shaozhu Chen, Department of Mathematics, Shandong University (Weihai), Weihai 264209, P. R. China

E-mail: szchen@sdu.edu.cn 